Article

\title{
Probiotic Characterization and Population Diversity Analysis of Gut-Associated Pediococcus acidilactici for Its Potential Use in the Dairy Industry
}

\author{
Hafsa Kanwal ${ }^{1}$, Alessandro Di Cerbo 2, , Freeha Zulfiqar ${ }^{1}$, Carla Sabia ${ }^{3}$, Amara Nawaz ${ }^{1}$, \\ Fariha Masood Siddiqui ${ }^{1}$, Muhammad Aqeel ${ }^{4}$ and Shakira Ghazanfar ${ }^{4}$ \\ 1 Department of Life Sciences, Abasyn University, Islamabad 44000, Pakistan; \\ hafsakanwal1588@gmail.com (H.K.); freehazulfiqar536@gmail.com (F.Z.); \\ amaranawaz613@gmail.com (A.N.); fariha.masood@abasynisb.edu.pk (F.M.S.) \\ 2 School of Bioscience and Veterinary Medicine, University of Camerino, 62024 Matelica, Italy \\ 3 Department of Life Sciences, University of Modena and Reggio Emilia, 41125 Modena, Italy; \\ carla.sabia@unimore.it \\ 4 National Agricultural Research Centre, National Institute of Genomics and Agriculture Biotechnology \\ (NIGAB), Park Road, Islamabad 45500, Pakistan; muhammadaqeel24@gmail.com (M.A.); \\ shakira_akmal@yahoo.com (S.G.) \\ * Correspondence: alessandro.dicerbo@unicam.it; Tel.: +39-0-737-403-466
}

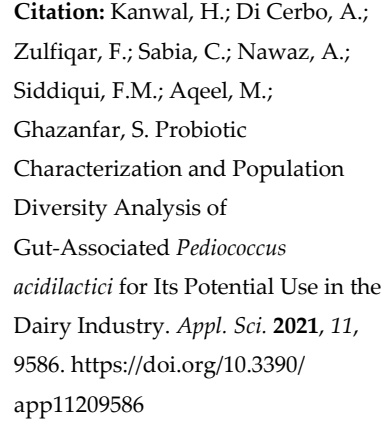

Academic Editor: Malgorzata Ziarno

Received: 13 September 2021

Accepted: 9 October 2021

Published: 14 October 2021

Publisher's Note: MDPI stays neutral with regard to jurisdictional claims in published maps and institutional affiliations.

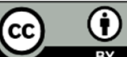

Copyright: (c) 2021 by the authors. Licensee MDPI, Basel, Switzerland. This article is an open access article distributed under the terms and conditions of the Creative Commons Attribution (CC BY) license (https://creativecommons.org/license s/by/4.0/).

\begin{abstract}
In recent years, gut-tailored probiotics have been proven to be beneficial for host health. Probiotic strains such as lactic acid bacteria (LAB) are known to exhibit antimicrobial activity, acting as natural substitutes for the regulation of foodborne pathogens. In the present study, a complete analysis, isolation, biochemical characterization, and molecular identification of Pediococcus acidilactici (NMCC-11) from Nili Ravi water buffalo (Bubalis bubalis) gut was carried out. NMCC-11 showed the best enzymatic potential, antimicrobial activity against known pathogenic strains, and survivability at a wide $\mathrm{pH}$ range $(\mathrm{pH} 4-\mathrm{pH} 6)$ out of all isolates. The isolates were screened for their antimicrobial activity against the five most infectious microbes such as Escherichia coli (ATCC 8739), Pseudomonas aeruginosa (ATCC9027), Staphylococcus aureus (ATCC6538), Listeria monocytogenes (ATCC13932), and Bacillus cereus (ATCC 11778) using the agar-well diffusion method. Moreover, after NMCC-11 isolation, a comparative diversity analysis against a variety of other randomly selected strains from around the world was carried out using $\mathrm{R}$ software. This study showed relatively low genetic diversity, which also contributed to the claim of the stability of this probiotic strain and its potential use as a starter culture and feed probiotic in the dairy industry. However, further studies are certainly warranted to determine its optimal dosage, time frame, and intake frequency.
\end{abstract}

Keywords: Pediococcus acidilactici; $16 \mathrm{~S}$ rRNA; probiotic; population genetic analysis; AMOVA; MSN; boxplot

\section{Introduction}

Probiotics are known to produce many vitamins (folates) and compounds (bacteriocins and low molecular mass molecules) that are able to promote a healthy digestive tract and trigger the immune system [1-4]. However, attention has now shifted to additional functions such as animal health promotion; disease prevention; animal performance enhancement, i.e., intake, weight gain, and milk yields; through oral application as additives in animal feeding.

Probiotic Gram-positive Pediococcus acidilactici is well-known for its use as supplement in treating constipation, diarrhea, stress, and immune response in birds and small animals; however, human trials are still limited [5]. The antipathogenic activity of 
the probiotic is an important factor for the selection of probiotics for the prevention of pathogenic infections and improved health of the organism. Pediococcus acidilactici has also been used for the production of potential bacteriocin known as "pediocin", which is used as a selective bacteriocin for controlling Listeria monocytogenes in maize silages [6].

Pediococcus acidilactici was selected among the isolates from the gut of Nili Ravi water buffalo (Bubalis bubalis) and partially sequenced using DNA encoding for 16S ribosomal RNA (rRNA; part of the $30 \mathrm{~s}$ subunit of the ribosome), for its potential application in the dairy industry as a feed probiotic and starter culture for various dairy products [7].

The gene coding for $16 \mathrm{~S}$ rRNA is highly conserved is, therefore, used in phylogenic reconstruction due to the slow evolution rates of this gene region [8]. In this study, 16S rRNA was also used for the calculation of the genetic distance, molecular variance, and other indices of the isolates for comparison of the results from probiotic species that were isolated from various sources around the globe.

For biologists attempting to understand community structure, the ability to evaluate diversity in this manner is a valuable resource. Understanding the pathways of evolution in bacteria by targeting various genes creates a better exposure toward biodiversity resulting from single base-pair changes.

The aim of the work was to isolate and biochemically characterize a potential probiotic strain from the gut of the domestic Nili Ravi water buffalo breed in Pakistan and conduct a diversity study using the 16S rRNA gene to study the diversity and evolution pattern by comparing it to randomly selected strains from around the world for its potential use in the dairy industry.

\section{Materials and Methods}

\subsection{Sample Collection}

Fresh fecal samples (10 g) were collected from 18 nursing Nili Ravi water buffalo using sterile gloves from the Livestock Research Station, National Agricultural Centre (NARC), Islamabad, Pakistan. The samples were collected in sterile containers (Deltalab, Spain) early in the morning and immediately transferred to the laboratory for microbiological analysis.

Research samples were tested for the presence of potential probiotic strains. The strains were checked for their probiotic activity according to the standardized microbiological technique proposed by Khan et al. [7].

\subsection{Isolation of Bacteria}

For dilution, $1 \mathrm{~g}$ of the sample was combined with $10 \mathrm{~mL}$ of saline phosphate buffer and mixed for 15-20 min using the vortex. For the growth and isolation of beneficial bacteria from the diluted samples, DeMan, Rogosa, and Sharpe (MRS) agar media (Oxoid, Karachi, Pakistan) were used. A total of $100 \mu \mathrm{L}$ of each diluted sample was spread on MRS agar and then incubated for $24-28 \mathrm{~h}$ at $37^{\circ} \mathrm{C}$.

\subsection{Morphological and Biochemical Characterization of Isolates}

Colonies from incubated Petri dishes were sub-cultivated to obtain pure isolates. The morphological identification of the pure isolates was also used for various biochemical studies (Figure 1). Bacterial colonies indicated as catalase- and oxidase-negative were further were inoculated in MRS medium containing $200 \mu \mathrm{g} / \mathrm{ml}$ lactic acid according to the concentration of lactic acid in buffalo rumen [9]. The bacterial isolates were cultured for $170 \mathrm{~h}$ at $28^{\circ} \mathrm{C}$, with the size of the bacterial colonies noted every $24 \mathrm{~h}$ during that time. The strain with the highest cellular viability, NMCC-11, was chosen for antibacterial testing and further probiotic analysis. 


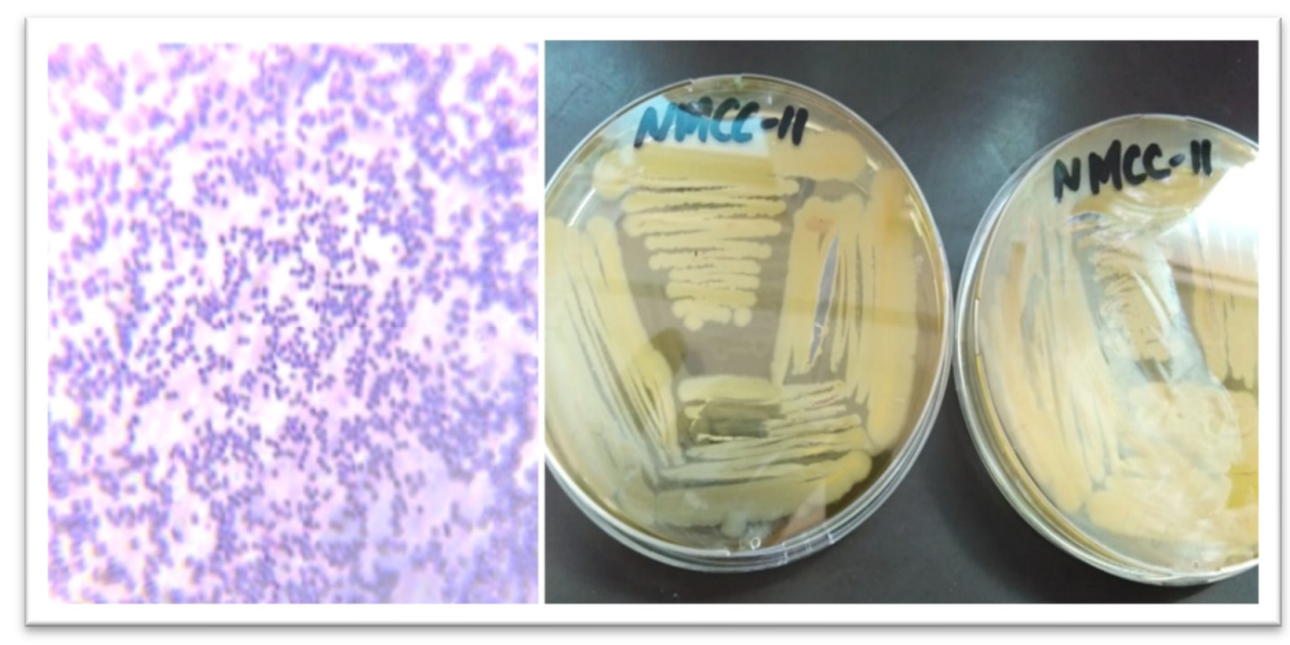

Figure 1. Gram-Positive Pediococcus under a light microscope that was obtained from cattle feces (left panel). Typical colony characteristics of the NMCC-11 isolates grown on an MRS agar medium (right panel).

\subsection{Determination of Probiotic and Antibacterial Potential}

\subsubsection{Enzymatic Potential}

Medium reported by Manhar et al. was used to detect the isolates cellulolytic potential [10]. Briefly, starch $(1 \mathrm{~g})$ and nutrient agar $(1 \mathrm{~g})$ were dissolved in distilled water $(100 \mathrm{~mL})$ to grow a pure bacterial culture and to assess amylolytic activity. The isolate's proteolytic activity was calculated using the method defined by Marlida et al. [11].

\subsubsection{Acid Tolerance}

The acid tolerance was calculated by preparing different $\mathrm{pH}$ levels of the MRS broth tubes ( $\mathrm{pH} 4.0,5.0$, and 6.0). A measure of $1 \mathrm{ml}$ of pure fresh culture was placed into MRS broth and aerobically incubated at $40^{\circ} \mathrm{C}$ for $48 \mathrm{~h}$. Total viable counts were determined at $0 \mathrm{~h}, 2 \mathrm{~h}$, and $4 \mathrm{~h}$ of incubation and the viable number of bacteria were enumerated by pour plate counts (CFU/mL) [12].

\subsubsection{Antibiotic Resistance and Antimicrobial Activity}

The susceptibility of LAB isolate to antibiotics was tested using the disc diffusion method [13]. The isolate was anaerobically grown at $30{ }^{\circ} \mathrm{C}$ in MRS broth for $24 \mathrm{~h}$. The antimicrobial activity of the bacterial strains was tested using pathogenic strains such Escherichia coli (ATCC 8739), Pseudomonas aeruginosa (ATCC 9027), Staphylococcus aureus (ATCC 6538), Listeria monocytogenes (ATCC 13932), and Bacillus cereus (ATCC 11778) according to Ghazanfar et al. [14].

\subsubsection{DNA Extraction and Amplification}

For genomic DNA extraction, purified bacterial colonies of NMCC-11 strain were suspended in $20 \mathrm{~mL}$ of Tris EDTA buffer in micro-PCR strips. The cells were lysed by heating at $95{ }^{\circ} \mathrm{C}$ for $10 \mathrm{~min}$, and cellular debris was removed by centrifugation at $4032 \times \mathrm{g}$ for $3 \mathrm{~min}$. The supernatant obtained after discarding the pellet served as a DNA template for 16S rRNA gene amplification [14]. The 16S rRNA gene of the putative LAB bacteria was amplified using Takara Pre-Mix Ex-Taq [15].

\subsection{Sequencing Analysis}

The amplified 16S rRNA gene of the strain was then partially sequenced by Sanger dideoxy sequencing targeting V3 and V4 regions [16]. Sequences were screened for 
chimeras by using Greengenes. The sequence was then submitted to the National Center for Biotechnology Information (NCBI) under a unique accession number [17]. The sequence in the Fast Adaptive Shrinkage Threshold Algorithm (FASTA) format was retrieved from NCBI using the accession number and submitted to the Basic Local Alignment Search Tool (BLAST) tool to calculate the percentage identity of sequences using the NCBI database of known sequences. The BLAST analysis results were subjected to multiple sequence alignment using the Molecular Evolutionary Genetics Analysis (MEGA)-X [18].

\subsection{Statistical Analysis}

The population genetic analysis included genotypic diversity measures, genetic distances native organization, handling of population hierarchies, and clone correction using R statistical software (Version 4.0.2, R Foundation for Statistical Computing, Murray Hill, NJ, USA) and its poppr and xlxs libraries [19].

The $\mathrm{R}$ statistical software that was used allowed us to rapidly view heterozygosity, evenness, and linkage indices to help decide further paths of investigation for a specific dataset. It also offered critical statistics about the rareness and richness of selected species inside a community by calculating diversity and relative indices.

This process involved the use of allele frequencies and SNP data. For cluster analysis, the Minitab 16 (Minitab Ltd., Coventry, UK) was used to create a dendrogram. The expected/observed heterozygosity, number of alleles per locus, and subpopulation statistics were computed by $\mathrm{R}$ language citations (locus_table, $\mathrm{mlg}$, nei.dist, and plot_poppr_msn) using poppr package [20]. The strain and their accession numbers were used as a template/sample hence they are not present in final graphs; however, they form a baseline if added.

The analysis of molecular variance (AMOVA) was used to find within-sample variance (error). This was performed in $\mathrm{R}$ by splitting the genotypes across all loci to create multiple haplotypes, which helped to calculate the within-sample distances and incorporate them into the model.

Data concerning NMCC-11 viability at three different time intervals and different $\mathrm{pH}$ values were analyzed using GraphPad Prism 8 software (GraphPad Software, Inc., La Jolla, CA, USA). All data are presented as the means \pm standard deviation and were first checked for normality using the D'Agostino-Pearson normality test. An ordinary one-way analysis of variance (ANOVA) followed by Tukey's multiple comparisons test was used to compare differences between different $\mathrm{pH}$ values within a time interval. A $p$ value $<$ 0.05 was considered significant.

\section{Results}

The NMCC-11 strain was classified as a probiotic as a result of in vitro morphological, biochemical, and probiotic studies (Figure 1). Strain NMCC-11 showed a 100\% homology with Pediococcus acidilactici, according to NCBI results (Table 1). 
Table 1. Biochemical and morphological characterization of Pediococcus acidilactici and its identification based on the $16 \mathrm{~S}$ rRNA gene sequence, along with the published accession numbers in the DNA database.

\begin{tabular}{|c|c|c|c|}
\hline \multicolumn{2}{|c|}{ Colony Morphological Characterization } & \multicolumn{2}{|c|}{ Identification Based on 16S rRNA Sequence } \\
\hline Gram staining & Positive cocci & \multirow{2}{*}{ Strain ID } & \multirow{2}{*}{ NMCC-11 } \\
\hline Shape & Circular & & \\
\hline Surface & Smooth & \multirow{2}{*}{ Probiotic strain genus } & \multirow{2}{*}{ Pediococcus acidilactic } \\
\hline Size & $>0.098 \mathrm{~mm}$ & & \\
\hline Color & White & \multirow{2}{*}{ Length of $16 \mathrm{~S}$ rRNA gene } & \multirow{2}{*}{$634 \mathrm{bp}$} \\
\hline Margin & Entire & & \\
\hline Elevation & Raised & \multirow{2}{*}{ Accession number } & \multirow{2}{*}{ MK606112 } \\
\hline Biochemical cha & rization & & \\
\hline Catalase & Negative & \multirow{2}{*}{ Closely related published taxa } & \multirow{2}{*}{ Pediococcus acidilactici } \\
\hline Oxidase & Negative & & \\
\hline Glucose fermentation & Positive & Sequence similarity & $99.21 \%$ \\
\hline
\end{tabular}

The NMCC-11 isolate was confirmed as a Gram-positive coccus using sample microscopy (Figure 1), and after, negative catalase and oxidase tests were established (Table 1). For additional microbiological assessment, the viability of strain under various conditions was tested.

The pure isolate was grown on MRS agar medium at $37^{\circ} \mathrm{C}$ for $24 \mathrm{~h}$, which resulted in circular, white, and smooth-textured colonies (Figure 1). The identification of presumed probiotic bacterial strains by molecular means is a critical step in gaining access to and assessing the safety of microorganisms of animal origin. The isolate was provisionally identified as Pediococcus acidilactici after molecular identification employing the $16 \mathrm{~S}$ rRNA gene (Table 1).

The phylogenetic tree in Figure 2 shows the evolutionary history of Pediococcus acidilactici.

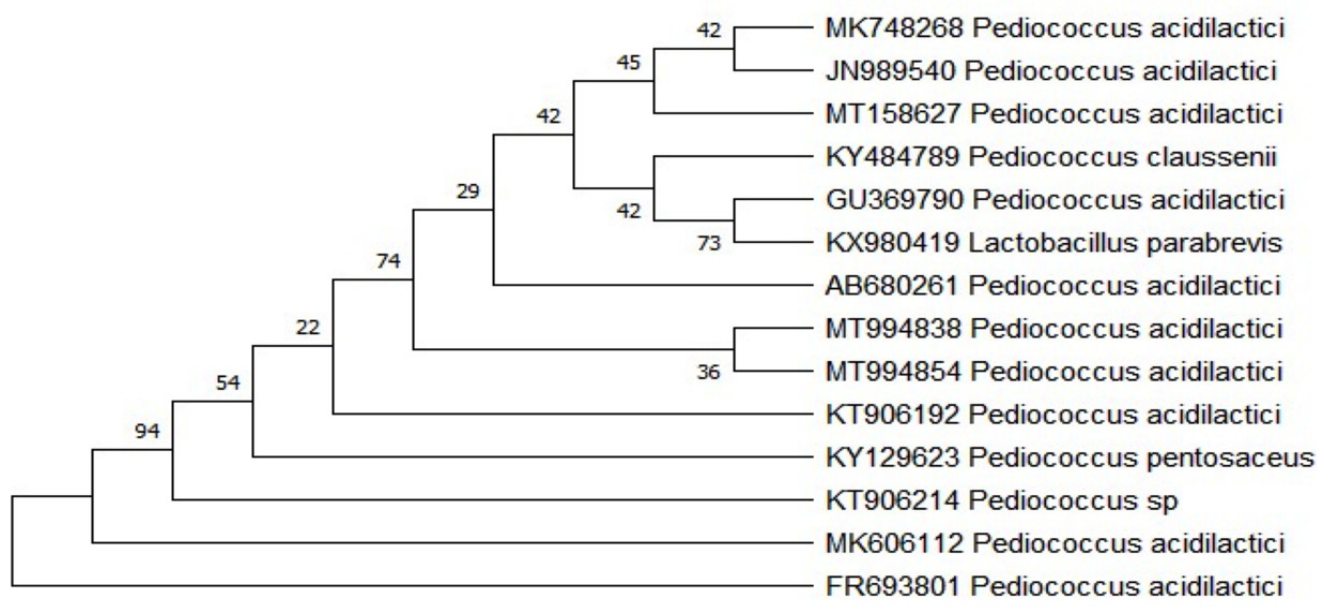

Figure 2. Schematic representation of the phylogenetic tree showing closely related species interfered from the 16S rRNA gene.

The phylogenetic tree was constructed using MEGA-X from the 16S rRNA gene sequence using the neighbor-joining algorithm and shows the closely related species. The types of strains used for comparison are given along with their accession numbers (Figure 2). The NMCC-11 strain was grown at different temperatures on MRS agar and $\mathrm{NaCl}$ 
concentration in M-17 broth for $24-48 \mathrm{~h}$. The amylolytic, proteolytic, and cellulolytic activity were tested along with bacteriocin production (Table 2).

Table 2. Viability of NMCC-11 at different temperatures and $\mathrm{NaCl}$ concentration and its enzymatic potential.

\begin{tabular}{ccccc}
\hline Temperature & & NaCl Concentration in MRS Broth & Enzymatic Potential \\
\hline $5-10^{\circ} \mathrm{C}$ & - & $0.5 \%$ & ++ & Amylolytic activity \\
$30-35{ }^{\circ} \mathrm{C}$ & + & $2 \%$ & - & Proteolytic activity \\
$37^{\circ} \mathrm{C}$ & ++ & $3 \%$ & - & Cellulolytic activity \\
$45^{\circ} \mathrm{C}$ & + & $6.5 \%$ & - & Bacteriocin production \\
$60^{\circ} \mathrm{C}$ & - & $10 \%$ & - & + \\
\hline
\end{tabular}

$(-)$ indicates no growth/activity, (+) indicates positive growth/activity, (++) indicates extensive growth rate/activity.

The NMCC-11 strain demonstrated optimum growth at temperatures $37^{\circ} \mathrm{C}$ and $0.5 \%$ of $\mathrm{NaCl}$; however, the viability of this probiotic bacterial strain was estimated at different temperatures and concentrations of $\mathrm{NaCl}$ (Table 2). Positive results were also achieved for amylolytic, proteolytic, and cellulolytic activity and bacteriocin production. The NMCC11 strain also demonstrated tolerance to a wide $\mathrm{pH}$ range of 4 to 6 (Figure 3 ).
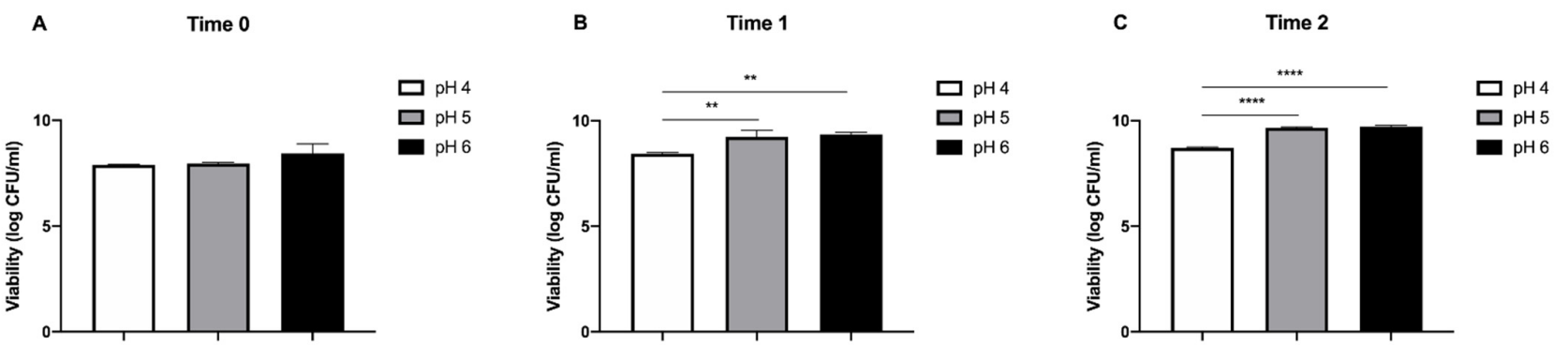

Figure 3. Schematic representation of the NMCC-11 viability (mean log CFU/mL \pm SD) at (A) the beginning of the experiment, (B) after 2 hours and (C) after 4 hours and at different $\mathrm{pH}$ values; ${ }^{* *} p<0.01,{ }^{* * * *} p<0.0001$.

No significant differences, in terms of bacterial viability, among different $\mathrm{pH}$ levels were observed at Time 0 (Figure 3A). Conversely, after $2 \mathrm{~h}$ (Time 1), a significant increase in bacterial viability (from $8.45 \pm 0.04 \log \mathrm{CFU} / \mathrm{mL}$ to $9.23 \pm 0.31$ and $9.35 \pm 0.1 \log \mathrm{CFU} / \mathrm{mL}$, ** $p<0.01$ ) was observed at $\mathrm{pH} 5$ and 6 compared to $\mathrm{pH} 4$, respectively.

A similar trend was observed after $4 \mathrm{~h}$ (Time 2); a significant increase in bacterial viability (from $8.70 \pm 0.05 \log \mathrm{CFU} / \mathrm{mL}$ to $9.65 \pm 0.05$ and $9.72 \pm 0.07 \log \mathrm{CFU} / \mathrm{mL},{ }^{* * *} p<$ 0.0001 ) was observed at $\mathrm{pH} 5$ and 6 compared to $\mathrm{pH} 4$, respectively.

By producing essential organic acids, hydrogen peroxide, and certain antimicrobial substances and bacteriocins, NMCC-11 also showed a good antimicrobial effect against Pseudomonas aeruginosa (ATCC 9027), Staphylococcus aureus (ATCC 6538), Listeria monocytogenes (ATCC 13932), and Bacillus cereus (ATCC 11778) (Table 3).

Table 3. Antibacterial behavior of bacterial strain against five pathogens as candidates for cattle probiotics.

\begin{tabular}{cccccc}
\hline Strain & \multicolumn{4}{c}{ Test Pathogen } \\
\hline Pediococcus acidilactici & Escherichia coli & Pseudomonas & Staphylococcus & Listeria & Bacillus \\
(NMCC-11) & - & aeruginosa & aureus & monocytogenes & cereus \\
& - & ++ & ++ & ++ & ++ \\
\hline
\end{tabular}

$(-)$ indicates no antimicrobial activity, (++) indicates strong antimicrobial activity.

Pediococcus acidilactici was able to inhibit the growth of the majority of the test organisms. Additionally, when monitoring the antimicrobial impact of Pediococcus acidilactici on pathogen growth, viable cells of Pseudomonas aeruginosa, Staphylococcus 
aureus, Listeria monocytogenes, and Bacillus cereus were not recorded during the period of study, confirming the efficacy of the antimicrobial substances and bacteriocins produced by NMCC-11. The absence of transferable resistance to therapeutic antibiotics is important for a probiotic strain. Therefore, the sensitivity of the majority of strains enables probiotics for their safe use in a variety of human and animal products [21,22]. For this purpose, the hemolytic function and antibiotic resistance of NMCC-11 have been determined (Table 4).

Table 4. Antibiotic resistance profile of NMCC-11 for cattle probiotic against common antibacterial compounds.

\begin{tabular}{ccc}
\hline Antibiotic & Zone of Inhibition & Mean Zone Diameter \\
\hline Ampicillin (5 ug) & $(\mathrm{S})$ & $30 \mathrm{~mm}$ \\
Penicillin G (10 ug) & $(\mathrm{S})$ & $22 \mathrm{~mm}$ \\
Lincomycin $(10 \mathrm{ug})$ & $(\mathrm{R})$ & $00 \mathrm{~mm}$ \\
Cloxacillin $(10 \mathrm{ug})$ & $(\mathrm{I})$ & $17 \mathrm{~mm}$ \\
Streptomycin $(10 \mathrm{ug})$ & $(\mathrm{I})$ & $16 \mathrm{~mm}$ \\
Erythromycin $(15 \mathrm{ug})$ & $(\mathrm{S})$ & $30 \mathrm{~mm}$ \\
Tetracycline $(30 \mathrm{ug})$ & $(\mathrm{S})$ & $31.5 \mathrm{~mm}$ \\
Oxytetracycline $(10 \mathrm{ug})$ & $(\mathrm{S})$ & $20 \mathrm{~mm}$ \\
\hline
\end{tabular}

Zone of inhibition: resistant (R): $\leq 15 \mathrm{~mm}$, intermediate (I) $16-20 \mathrm{~mm}$, susceptible (S): $\geq 21 \mathrm{~mm}$.

Pediococcus acidilactici NMCC-11 was shown to be susceptible to Ampicillin $(5 \mu \mathrm{g})$, Penicillin $(10 \mu \mathrm{g})$, Erythromycin $(15 \mu \mathrm{g})$, Tetracycline (30 ug), and Oxytetracycline (10 $\mu \mathrm{g})$ antibiotics, but resistant to Lincomycin $(10 \mu \mathrm{g})$ with intermediate sensitivity to Cloxacillin $(10 \mu \mathrm{g})$ and Streptomycin $(10 \mu \mathrm{g})$. This finding indicates the presence of the Lincomycin resistance gene in the genome.

These antibiotic markers help in understanding the suitability of the strain to use as a dietary supplement. Genetic analysis of biodiversity provided insight into details about the different parameters of diversity analysis (Figure 4).
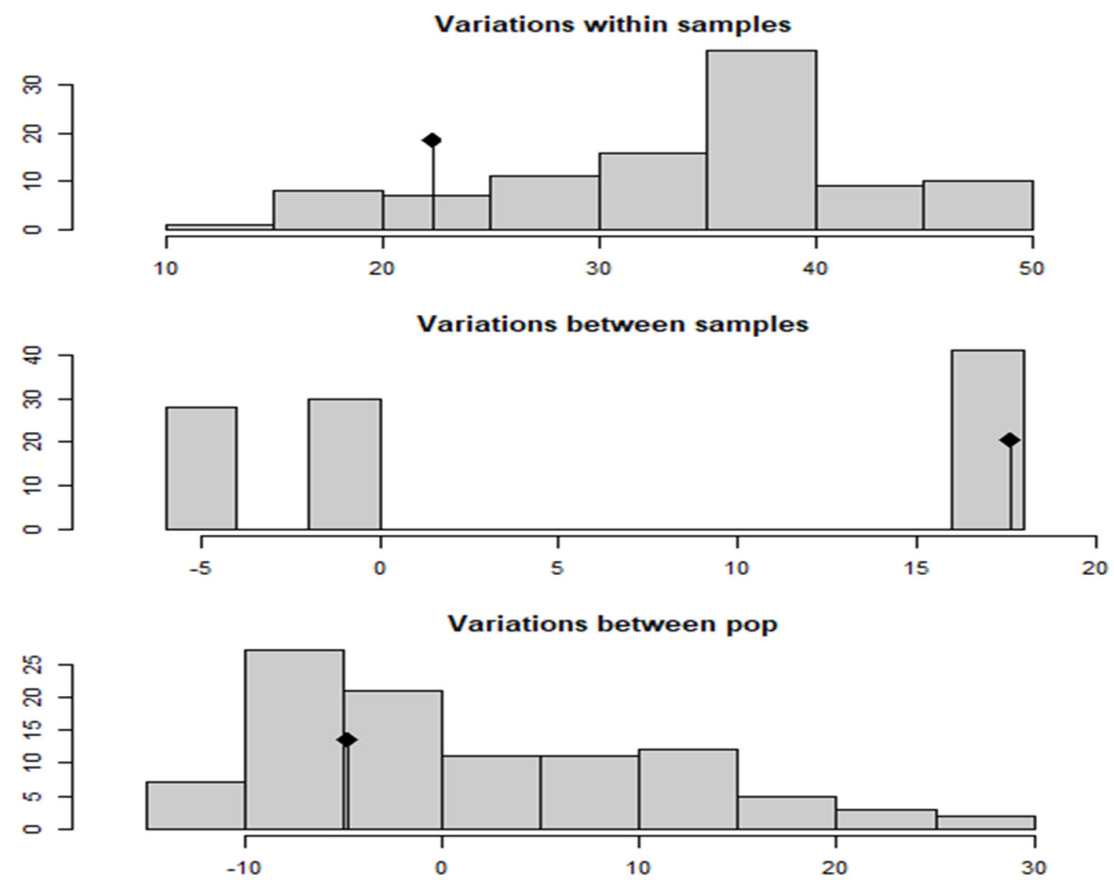

Figure 4. Schematic representation of the AMOVA MK606112 histograms shows the distribution of the randomized strata, and the black line shows the observed data. 
The black line represents the observed data while the bars of the histograms represent the randomized strata's distribution. For this study, the null hypothesis was the HardyWeinberg equilibrium (i.e., if the $p$-value is less than or equal to a significant level/cut-off (0.05), then the null hypothesis is rejected). Given that the observed data did not fall within the expected distribution from the permutation, Figure 4 shows the horizontal axis as genetic distance values, highlighting substantial population differentiation at one level out of all three levels $(p=0.01)$. No significant difference was observed within samples and between subpopulation levels $(p>0.05)$.

Values of AMOVA (Table 5) showed the expected and observed heterozygosity, as well as polymorphism information of alleles, which revealed significant population structure at one out of three levels of population strata.

Table 5. AMOVA results show significant variation between samples based on $p$-value.

\begin{tabular}{ccccc}
\hline Test & Obs & Std.Obs & Alter & $p$-Value \\
\hline Variation within samples & 22.291667 & -1.4080075 & less & 0.13 \\
Variation between samples & 17.637500 & 1.1759021 & greater & 0.01 \\
Variation between sub pop & -4.784459 & -0.6088021 & greater & 0.65 \\
\hline
\end{tabular}

A distance matrix was used to generate a minimum spanning network (MSN) of selected populations. Each node represented a different multilocus genotype (MLG) (Figure 5). The number of individuals and population membership was represented by node sizes and colors, respectively. The color and thickness of the edges were proportional to Bruvo's distance.

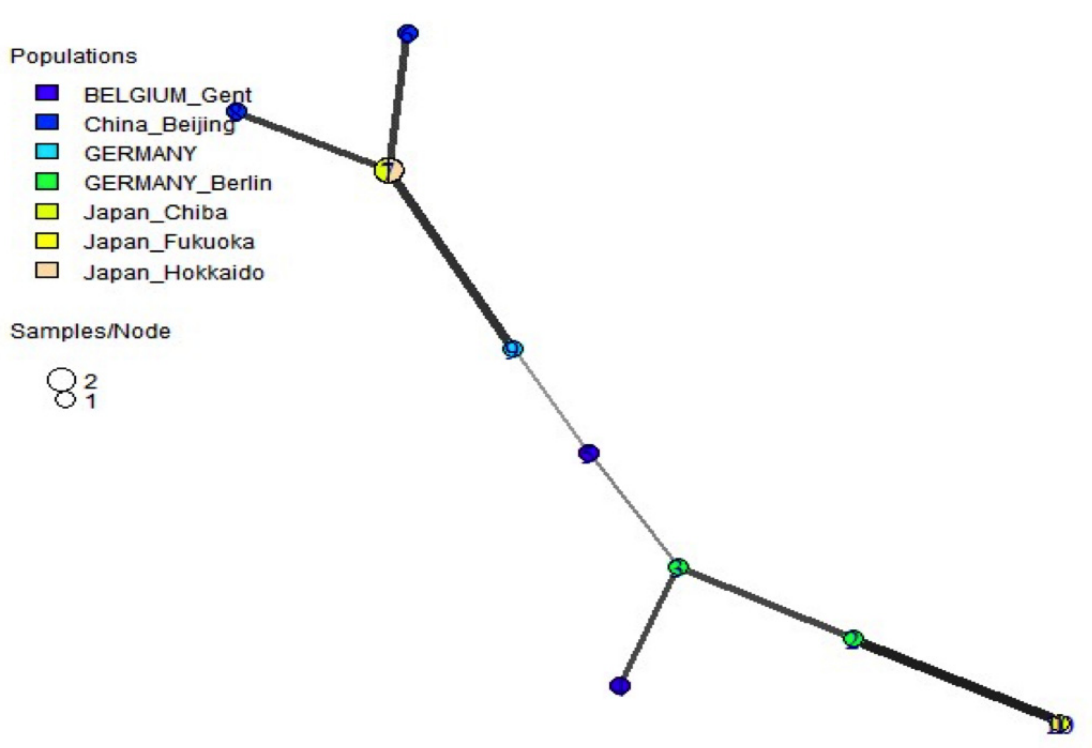

Figure 5. Schematic representation of the minimum spanning network representing 7 simulated populations.

Clustering allowed the grouping of data based on their similarity; the results can be seen as a dendrogram (Figure 6). 


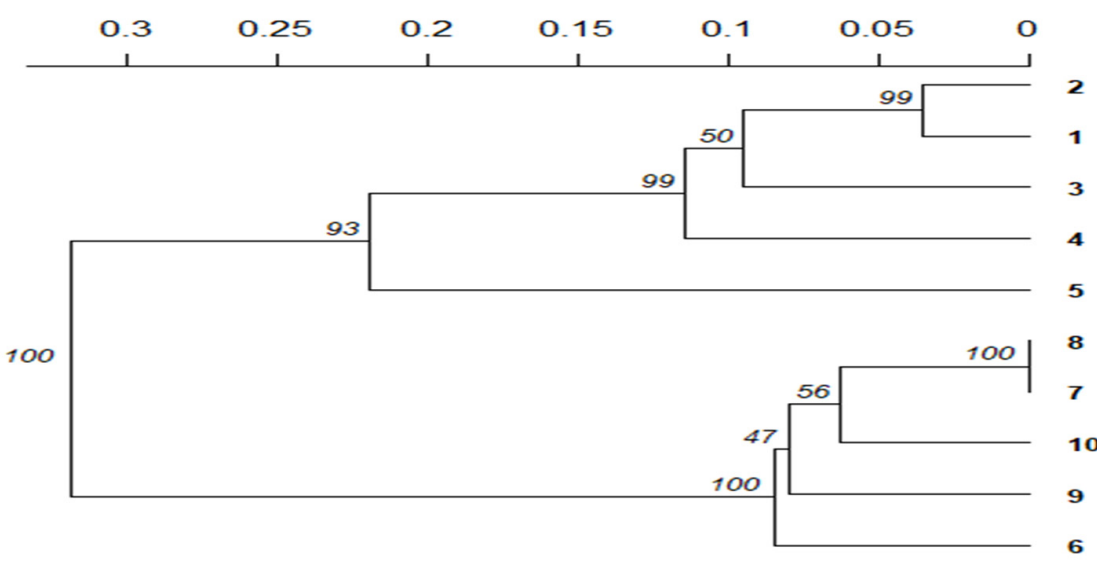

Figure 6. Schematic representation of the cluster dendrogram based on Nei's coefficient with 10 tips and 9 internal nodes.

Genetic distance is a metric for comparing genetic differences across populations or closely related species and it is typically calculated by combining allele frequency data from many populations' loci. The rooted unweighted pair group method with arithmetic mean (UPGMA), bottom-up hierarchical cluster diagram was built using Nei's coefficient of similarity, which increases in proportion to the divergence time (Figure 6).

The population genetics parameters in diversity measures include both genotypic abundance and richness (Figure 7), taking equal sample sizes and a standard error (0) into account in all populations. 


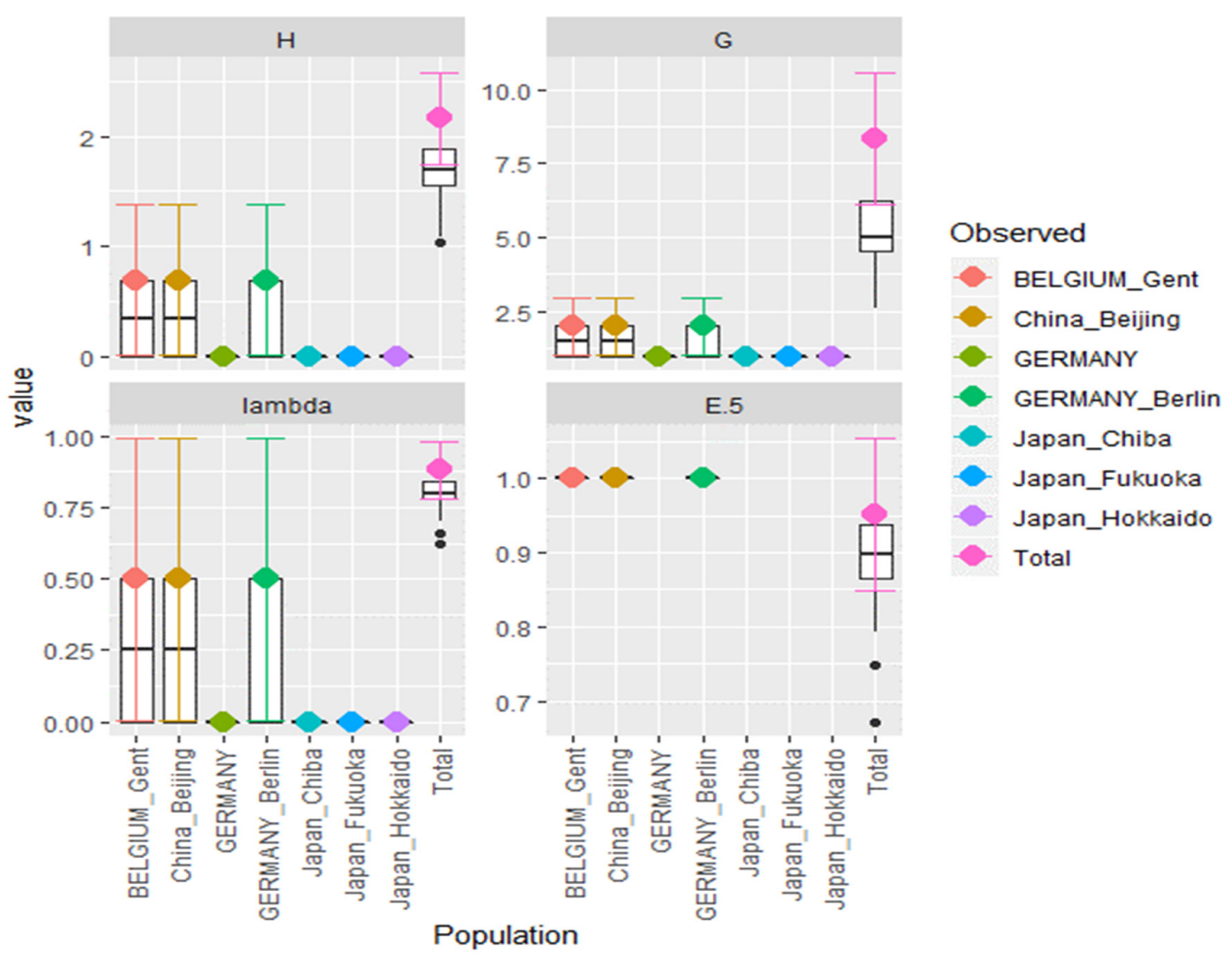

Figure 7. Schematic representation of the diversity box plot representing: H (Shannon's index), G (inverse Simpson's index), lambda (Simpson index), and E5 (evenness).

The diversity box plots were created using the values in the diversity statistics table that depicted each population, as categorized by statistics, and provided by the values of diversity indices, i.e., $\mathrm{H}$ (Shannon's index), G (inverse Simpson's index), lambda (Simpson index), and E5 (evenness) in the table, revealing that the population was more diverse with higher MLGs.

\section{Discussion}

Several probiotic supplements are available commercially on the market, but their probiotic ability in local breed feed is unknown. Therefore, indigenous probiotic organisms from local animal breeds need to be studied. The probiotic strains of the same ecological niche isolated from the native organism may be more compatible with gut microbiota [23]. The present experiment was carried out to distinguish probiotics from the breed of domestic Nili Ravi water buffalos in Pakistan and to investigate its variety worldwide.

In this sense, the NMCC-11 strain was classified as a probiotic as a result of in vitro morphological, biochemical, and probiotic studies showing a $100 \%$ homology with Pediococcus acidilactici, according to NCBI results [17].

Pediococcus acidilactici was also isolated from cattle gut by Rodriguez-Palacios et al. [24]. Low-pH resistance is a noticeable feature of probiotic bacteria since they need to withstand the acidic condition of the stomach to enter the intestine. The $\mathrm{pH}$ value in the stomach can be as low as 1.0, but the $\mathrm{pH}$ value between 3.0 and 4.0 is often favored during in vitro assays because the viability of bacterial strains decreases below $\mathrm{pH} 3.0$ [25]. The NMCC-11 strain demonstrated optimum growth at temperatures $37^{\circ} \mathrm{C}$ and $0.5 \%$ of NaCl; however, its viability was estimated at different temperatures and concentrations of $\mathrm{NaCl}$. 
The viable bacterial count on MRS media at $\mathrm{pH} 5$ to 5.5 was in line with previous observations [26]. Probiotic strain's enzymatic behavior is also a critical phenomenon that increases the efficiency of feed utilization [23]. The NMCC-11 strain showed strong proteolytic activity; positive results were also achieved for amylolytic, cellulolytic activity, and bacteriocin production. The amylase enzyme is generated by a hydrolyzing bacterial strain that ferments and transforms starch into lactic acid [27]. As stated in previous findings, the isolated NMCC-11 strain also showed cellulolytic activity. In agreement with this, researchers also found that Pediococcus acidilactici had a positive enzymatic potential [28]. For unique probiotics, antimicrobial activity is a very important feature.

The measures of hydrophobicity and auto-aggregation are representative of the adhesive ability for the selection of probiotics. In the current analysis, as also stated by Osmanagaoglu et al. [29], the NMCC-11 isolates displayed strong hydrophobicity and auto-aggregation properties; the characteristics of the microbial surface were noted to see the interactions between bacteria and the interface. The hemolytic function and antibiotic resistance of NMCC-11 have been determined from a safety standpoint, which revealed its resistance to Lincomycin and sensitivity to five other antibiotics [30].

Genetic diversity is critical in the evolution of an organism. Microbial phenotypes are geologically ancient and have been preserved via stabilizing selection, although microbial species together reflect significant genetic diversity due to enormous absolute population numbers, a lack of allopatric speciation, and low extinction rates [31].

Different indices of diversity were applied using $\mathrm{R}$ software on the randomly selected strains from around the world. Among different indices of diversity, AMOVA revealed significant population differentiation at one out of all three levels because the observed data did not fall inside the expected distribution from the permutation [20]. The hue and thickness of the edges were proportional to Bruvo's distance in the minimal spanning network (MSN), which used a distance matrix for the selected populations representing various multilocus genotypes at each node [32]. Nei's genetic distance, which assumes that mutation and genetic drift cause the genetic differences if the rate of amino acid substitution is constant per year or generation, was used to create the rooted UPGMA bottom-up hierarchical cluster diagram [20].

The multilocus genotypes (MLG) revealed that the genotypic richness is certainly greater in three populations compared to the others. Three genotypic diversity measures were employed by poppr; comparing the results of these populations, the $\mathrm{H}$ (ShannonWiener Index) was lower for the populations and the G (aka inverse Simpson's index) was greater, which shows that diversity is higher in the populations with higher MLGs. Note that lower H (Shannon-Wiener Index) values indicate more diversity [20]. Keeping in mind that the overall MLG count was lower, the diversity pattern indicated a slow evolution rate that can be an indicator for the stability of this strain.

From the study findings, it is possible to infer that Pediococcus acidilactici NMCC-11 has the potential to be used as a probiotic microorganism in feed and starter culture and as a viable source to generate safer food to overcome some major challenges faced by the dairy industry. The majority of probiotic strains are known to have a recommended dose of $10^{9} \mathrm{CFU} / \mathrm{kg}$, applied orally as an additive in mixed feed at a frequency intake of 10 to 20 times per day; however, further studies are certainly warranted to investigate the precise dosage, frequency of intake, and time frame [32].

\section{Conclusions}

The 16S rRNA gene of a potential probiotic isolate NMCC-11 confirmed it as Pediococcus acidilactici, a strain renowned for its probiotic activity. The evaluation of probiotic activity revealed NMCC-11 had the best activity in its ability to survive in a wide $\mathrm{pH}$ range, antimicrobial activity against four main pathogens, enzymatic activity, bacteriocin production, and antibiotic resistance, thus reinforcing its potential application as a feed probiotic and starter culture in the dairy industry. 
Comparative diversity analysis of strains using $\mathrm{R}$ software against randomly selected strains from around the world revealed an overall low diversity and a notable population structure, which is a significant determinant of the durability of the strain.

The findings of this study could be used to further investigate the beneficial health impacts of Pediococcus acidilactici NMCC-11 on animal health as well as the quality of various products in the dairy industry.

Author Contributions: Conceptualization, H.K.; methodology, H.K.; software, M.A.; validation, H.K., M.A., and S.G.; formal analysis, H.K. and A.D.C.; investigation, H.K., A.N., and F.Z.; resources, S.G. and M.A.; data curation, H.K. and M.A.; writing-original draft preparation, H.K. and A.N.; writing - review and editing, H.K., A.D.C., and C.S.; visualization, H.K., M.A., and C.S.; supervision, S.G. and F.M.S.; project administration, S.G. and F.M.S.; funding acquisition, S.G. and A.D.C. All authors have read and agreed to the published version of the manuscript.

Funding: This research received no external funding.

Institutional Review Board Statement: Operative procedures (faecal sample collection) were performed in compliance with the national and international regulations to ensure the animal welfare. Moreover, the recommendations of the ARRIVE guidelines in animal research were also consulted and considered.

Informed Consent Statement: Not applicable.

Data Availability Statement: The data presented in this study are available on request from the corresponding author.

Conflicts of Interest: The authors declare no conflict of interest.

\section{References}

1. Rossi, M.; Amaretti, A.; Raimondi, S. Folate Production by Probiotic Bacteria. Nutrients 2011, 3, 118-134. https://doi.org/10.3390/nu3010118.

2. Blaiotta, G.; Murru, N.; Di Cerbo, A.; Romano, R.; Aponte, M. Production of probiotic bovine salami using Lactobacillus plantarum 299v as adjunct. J. Sci. Food Agric. 2018, 98, 2285-2294. https://doi.org/10.1002/jsfa.8717.

3. Di Cerbo, A.; Palmieri, B. Review: The market of probiotics. Pak. J. Pharm. Sci. 2015, 28, 2199-2206.

4. Di Cerbo, A.; Palmieri, B.; Aponte, M.; Morales-Medina, J.C.; Iannitti, T. Mechanisms and therapeutic effectiveness of lactobacilli. J. Clin. Pathol. 2016, 69, 187-203. https://doi.org/10.1136/jclinpath-2015-202976.

5. Fijan, S. Microorganisms with Claimed Probiotic Properties: An Overview of Recent Literature. Int. J. Environ. Res. Public Health 2014, 11, 4745-4767. https://doi.org/10.3390/ijerph110504745.

6. Amado, I.R.; Fuciños, C.; Fajardo, P.; Pastrana, L. Pediocin SA-1: A selective bacteriocin for controlling Listeria monocytogenes in maize silages. J. Dairy Sci. 2016, 99, 8070-8080. https://doi.org/10.3168/jds.2016-11121.

7. Khan, S.I.; Din, A.; Ali, G.M.; Khan, S.I.; Arif, I.; Riaz, M.N.; Ghazanfar, S. Screening of Lactic Acid Bacteria for Their Use as Buffalo Probiotic. J. Anim. Plant Sci. 2020, 30, 1357-1365.

8. Woese, C.R.; Fox, G.E. Phylogenetic structure of the prokaryotic domain: The primary kingdoms. Proc. Natl. Acad. Sci. USA 1977, 74, 5088-5090. https://doi.org/10.1073/pnas.74.11.5088.

9. Van Nieuwenhove, C.P.; Oliszewski, R.; Gonzalez, S.N.; Chaia, A.B.P. Conjugated linoleic acid conversion by dairy bacteria cultured in MRS broth and buffalo milk. Lett. Appl. Microbiol. 2007, 44, 467-474. https://doi.org/10.1111/j.1472-765x.2007.02135.x.

10. Manhar, A.K.; Bashir, Y.; Saikia, D.; Nath, D.; Gupta, K.; Konwar, B.K.; Kumar, R.; Namsa, N.D.; Mandal, M. Cellulolytic potential of probiotic Bacillus Subtilis AMS6 isolated from traditional fermented soybean (Churpi): An in-vitro study with regards to application as an animal feed additive. Microbiol. Res. 2016, 186-187, 62-70. https://doi.org/10.1016/j.micres.2016.03.004.

11. Marlida, R.; Suprayudi, M.; Widanarni; Harris, E. Isolation, Selection and Application of Probiotic Bacteria for Improvement the Growth Performance of Humpback Groupers (Cromileptes altivelis). Int. J. Sci. Basic Appl. Res. 2014, 16, 364-379.

12. Hassanzadazar, H.; Ehsani, A.; Mardani, K.; Hesari, J. Investigation of antibacterial, acid and bile tolerance properties of lactobacilli isolated from Koozeh cheese. Veter Res. Forum Int. Q. J. 2012, 3, 181-185.

13. Olatunde, O.O.; Obadina, A.O.; Omemu, A.M.; Oyewole, O.B.; Olugbile, A.; Olukomaiya, O.O. Screening and molecular identification of potential probiotic lactic acid bacteria in effluents generated during ogi production. Ann. Microbiol. 2018, 68, 433-443. https://doi.org/10.1007/s13213-018-1348-9.

14. Shakira, G.; Qubtia, M.; Ahmed, I.; Hasan, F.; Anjum, M.; Imran, M. Effect of indigenously isolated Saccharomyces cerevisiae probiotics on milk production, nutrient digestibility, blood chemistry and fecal microbiota in lactating dairy cows. J. Anim. Plant Sci. 2018, 28, 407-420. 
15. Ghazanfar, S.; Anjum, M.; Azim, A.; Ahmed, I.I. Effects of Dietary Supplementation of Yeast (Saccharomyces Cerevisiae) Culture on Growth Performance, Blood Parameters, Nutrient Digestibility and Fecal Flora of Dairy Heifers. J. Anim. Plant Sci. 2015, 25, 53-59.

16. Coenye, T.; Vandamme, P. Intragenomic heterogeneity between multiple $16 \mathrm{~S}$ ribosomal RNA operons in sequenced bacterial genomes. FEMS Microbiol. Lett. 2003, 228, 45-49. https://doi.org/10.1016/s0378-1097(03)00717-1.

17. Ghazanfar, S.; Ali, G.M. Pediococcus acidilactici strain NMCC-11 16 S ribosomal RNA gene, partial sequence. 2019. Available online: www.ncbi.nlm.nih.gov/search/all/?term=MK606112 (accessed on 25/08/2021).

18. Kumar, S.; Tamura, K.; Nei, M. MEGA: Molecular Evolutionary Genetics Analysis software for microcomputers. Comput. Appl. Biosci. 1994, 10, 189-191. https://doi.org/10.1093/bioinformatics/10.2.189.

19. Kamvar, Z.N.; Brooks, J.C.; Grunwald, N.J. Novel R tools for analysis of genome-wide population genetic data with emphasis on clonality. Front. Genet. 2015, 6, 208. https://doi.org/10.3389/fgene.2015.00208.

20. Shoukat, S.; Anwar, M.; Ali, S.; Begum, S.; Aqeel, M.; Zia, M.; Shah, S.; Ali, G. Estimation of Morphological and Molecular Diversity of Seventy-Two Advanced Pakistani Cotton Genotypes Using Simple Sequence Repeats. Appl. Ecol. Environ. Res. 2020, 18, 2043-2056. https://doi.org/10.15666/aeer/1802_20432056.

21. Di Cerbo, A. Presencia de Antibióticos Inusual en el Gimnasio Entrenando Sujetos. Nutr. Hosp. 2014, 30, 395-398. https://doi.org/10.3305/NH.2014.30.2.7594.

22. Iseppi, R.; Di Cerbo, A.; Messi, P.; Sabia, C. Antibiotic Resistance and Virulence Traits in Vancomycin-Resistant Enterococci (VRE) and Extended-Spectrum $\beta$-Lactamase/AmpC-producing (ESBL/AmpC) Enterobacteriaceae from Humans and Pets. Antibiot. 2020, 9, 152. https://doi.org/10.3390/antibiotics9040152.

23. Ghazanfar, S.; Khalid, N.; Ahmed, I.; Imran, I.A.A.M. Probiotic Yeast: Mode of Action and Its Effects on Ruminant Nutrition. In Yeast Industrial Applications; Morata, A., Loira, I., Eds.; IntechOpen: Rijeka, Croatia, 2017. https://doi.org/10.5772/intechopen.70778.

24. Rodriguez-Palacios, A.; Staempfli, H.; Duffield, T.; Weese, J. Isolation of bovine intestinalLactobacillus plantarumandPediococcus acidilacticiwith inhibitory activity againstEscherichia coliO157 and F5. J. Appl. Microbiol. 2009, 106, 393-401. https://doi.org/10.1111/j.1365-2672.2008.03959.x.

25. Naeem, M.; Ahmed, I.; Ahmed, S.; Ahmed, Z.; Riaz, M.N.; Ghazanfar, S. Screening of cattle gut associated Bacillus strains for their potential use as animal probiotic. Indian J. Anim. Res. 2018. https://doi.org/10.18805/ijar.b-948.

26. Morandi, S.; Brasca, M.; Alfieri, P.; Lodi, R.; Tamburini, A. Influence of $\mathrm{pH}$ and temperature on the growth of Enterococcus faecium and Enterococcus faecalis. Lait 2005, 85, 181-192. https://doi.org/10.1051/lait:2005006.

27. Abbasiliasi, S.; Tan, J.S.; Bashokouh, F.; Ibrahim, T.A.T.; Mustafa, S.; Vakhshiteh, F.; Sivasamboo, S.; Ariff, A.B. In vitro assessment of Pediococcus acidilactici Kp10 for its potential use in the food industry. BMC Microbiol. 2017, $17,1-11$. https://doi.org/10.1186/s12866-017-1000-z.

28. Ho, J.C.K.; Sze, L.Y. Isolation, identification and characterization of enzyme-producing lactic acid bacteria from traditional fermented foods. Biosci. Horizons: Int. J. Stud. Res. 2018, 11, 11. https://doi.org/10.1093/biohorizons/hzy004.

29. Osmanağaoğlu, Ö.; Kiran, F.; Ataoğlu, H. Evaluation of in vitro Probiotic Potential of Pediococcus pentosaceus OZF Isolated from Human Breast Milk. Probiotics Antimicrob. Proteins 2010, 2, 162-174. https://doi.org/10.1007/s12602-010-9050-7.

30. Parvez, S.; Malik, K.; Kang, S.A.; Kim, H.-Y. Probiotics and their fermented food products are beneficial for health. J. Appl. Microbiol. 2006, 100, 1171-1185. https://doi.org/10.1111/j.1365-2672.2006.02963.x.

31. Fenchel, T.; Finlay, B.J. The diversity of microbes: Resurgence of the phenotype. Philos. Trans. R. Soc. B: Biol. Sci. 2006, 361, 19651973. https://doi.org/10.1098/rstb.2006.1924.

32. Kamvar, Z.N.; Tabima, J.F.; Grünwald, N.J. Poppr: An R package for genetic analysis of populations with clonal, partially clonal, and/or sexual reproduction. PeerJ 2014, 2, e281. https://doi.org/10.7717/peerj.281. 